P-110 DYING TO BE INVOLVED- A SURVEY OF WHAT MATTERS TO AN ACUTE HOSPITAL COMMUNITY

Clare Smith, Natalie Wright, Sue Dargan, Sinead Henderson. Ashford and St Peters NHS Foundation Trust, Chertsey, UK

\subsection{6/bmjspcare-2017-00133.109}

In 2009, the Dying Matters Coalition was set up to promote public awareness of dying, death and bereavement. The aim was to address society's lack of openness and to encourage people to talk about their wishes towards the end of their lives with friends, family and loved ones, including thinking about where they want to die.

A Dying Matters survey was conducted at St Peters Hospital (ASPH), a District General Hospital in Surrey, UK. The aim was to open the discussion about death and dying to the ASPH community and determine 'what matters' to ASPH about death and dying.

Methods Potential participants were all people (including patients, relatives and staff) entering the hospital during dying matters week (May 2016). These participants were approached to complete a short anonymous questionnaire and provided free text comments. Participants were able to choose any number of areas of care that 'mattered' to them.

Results One hundred and seventy-seven completed questionnaires were returned. One hundred and twenty nine (73\%) were from females, and the majority of respondents were aged between $25-64$ years old $(n=138,78 \%)$. The element of care with the greatest response was 'being involved in care decisions' ( $n=152,86 \%)$. 'Being with those who are important to you' and compassionate care were the next most important $(n=144,81 \%)$. Symptom control mattered for $60 \%$ $(n=106)$. Dying at home for $64 \%(n=114)$. Analysis of free text comments showed a positive response to the dying matters awareness survey.

Conclusion This survey shows it is possible to engage an acute hospital community in dying awareness discussions. Of interest is the large majority of participants felt 'being involved in care decisions' was the most important element of care. Patient involvement in care decisions through shared decision making is pivotal to getting dying right for all.

\section{P-111 PALLIATIVE CARE TRAINING FOR NON-SPECIALISTS: WHAT HAPPENS IN UK MEDICAL SCHOOLS, PRIMARY CARE AND HOSPITAL PRACTICE? HOW MIGHT DEFICITS BE ADDRESSED?}

\footnotetext{
1,2,3 Iain Lawrie, ${ }^{3,4}$ Steven Walker. ${ }^{1}$ The Pennine Acute Hospitals NHS Trust, Manchester, UK; ${ }^{2}$ University of Manchester, UK; ${ }^{3}$ Centre for Medical Education, University of Dundee, UK; ${ }^{4}$ Stgilesmedical London and Berlin'
}

\subsection{6/bmjspcare-2017-00133.110}

Background and purpose PC has gained prominence in an effort to deliver quality care for people with end of life needs. ${ }^{1}$ Non-specialist doctors provide the majority of such care. $^{2}$ Limited research suggests lack of preparedness and confidence for this role. ${ }^{3}$ Similarly, weaknesses may exist in PC teaching at some medical schools. ${ }^{4}$ Two parallel studies explore this further.

Methodology A survey of General Practitioners (GPs) and Hospital Doctors (HDs) examined education, knowledge and confidence in managing patients with PC needs. A second study surveyed UK medical school PC course organisers, focusing on teaching and organisation. Results were analysed for common themes.

Results Most GPs and HDs regularly saw patients with PC needs, and viewed PC positively. Self-assessment of competences was mixed. Participation in postgraduate PC education was low. Attending conferences does not change practice. Clinical attachments, shadowing opportunities, e-learning and textbooks are more likely to be beneficial.

Undergraduates receive increased PC teaching time with greater curriculum integration and wider use of assessment however variability and areas of weakness exist. Concerns expressed include insufficient placements, teachers and funding. Doubts existed whether courses deliver quality training, adequately prepare doctors to care for PC patients, or fulfil General Medical Council requirements.

Discussion and conclusion PC teaching at medical school may be insufficient to equip doctors to care for patients with PC needs. There is a need for increased postgraduate training for non-specialists. To be effective, such training should be learner-centred, involve clinical scenarios and experiential learning, and recognition of barriers to PC education.

\section{REFERENCES}

1. Riley J. A strategy for end-of-life care in the UK. BMJ 2008;337:934-934

2. Association for Palliative Medicine of Great Britain and Ireland. Curriculum for Undergraduate Medical Education. http://www.apmuesif.phpc.cam.ac.uk/index. php/apm-curriculum (accessed 7 July 2014).

3. Lloyd-Williams M, MacLeod RD. A systematic review of teaching and learning in palliative care within the medical undergraduate curriculum. Med Teach 2004;26:683-690.

4. Gibbins J, McCoubrie R, Forbes K. Why are newly qualified doctors unprepared to care for patients at the end of life? Med Educ 2011;45:389-399.

\section{P-112 IMPROVING ACUTE HOSPITAL CARE THROUGH PARTNERSHIP WORKING}

${ }^{1}$ Katherine Frew, ${ }^{1}$ Carole Duff, ${ }^{2}$ Gillian Watson, ${ }^{2}$ Kay Wood, ${ }^{2}$ Patricia Robson, ${ }^{1}$ Louise Whitfield, ${ }^{1}$ Hannah Hall, ${ }^{1}$ Leonie Armstrong. ${ }^{1}$ Northumbria Healthcare NHS FT, North Shields, UK; ${ }^{2}$ Marie Curie

\subsection{6/bmjspcare-2017-00133.111}

Background Northumbria Healthcare NHS FT has developed a novel partnership approach with Marie Curie to improve hospital liaison palliative care. This has increased the number of palliative care nursing staff from 2.8 to 9 WTE posts. This service expansion has coincided with the opening of Northumbria Specialist Emergency Care Hospital (NSECH). NSECH has been highlighted by NHS England as an example of good practice and, in line with the five year forward view, ${ }^{1}$ may be replicated in other areas of the country. The vision is to provide emergency care within NSECH and ongoing, non-acute, care on 'base-site' hospitals. It is imperative to assess the impact of such a development on the development of palliative care services.

Design This service evaluation considered patient characteristics and outcome for patients seen in NSECH over 6 months following its opening. Data were extracted from an existing palliative care database.

Results 492 patients were seen, with 1232 face to face patient contacts.

At first assessment $65 \%$ were in an unstable phase of illness; 24\% were deteriorating and 10\% were terminally ill.

Mean performance status (KPS) was 39\%. 